\title{
Electrochemical Measurements during In Situ Liquid-Electrochemical TEM Experiments
}

\author{
Eli Fahrenkrug${ }^{1}$, Daan Hein Alsem ${ }^{2}$, Norman Salmon ${ }^{2}$, and Stephen Maldonado ${ }^{1,3}$ \\ 1. Department of Chemistry, University of Michigan, Ann Arbor, MI, USA \\ 2. Hummingbird Scientific, Lacey WA, USA \\ 3. Program of Applied Physics, University of Michigan, Ann Arbor, MI, USA
}

Electroanalytical measurements performed inside the transmission electron microscope (TEM) are becoming more common and are used to study a wide range of electrochemical reaction-systems at the nanoscale [1-8]. This approach has already started to produce new insights on the dynamics and structural changes during processes as lithium ion insertion/extraction [8], metal nucleation during electrodeposition [1], dendrite formation [3], and metal corrosion [6].

Despite the power of this approach, liquid-electrochemical TEM results can be challenging to interpret. Several potential artefacts can be identified here that could make this challenging:

- The influence of the electron (e-) beam used in TEM for imaging can induce (unwanted) reactions in liquid electrolytes.

- Electrochemical data recorded in a TEM can be influenced by either the thin liquid layer required to allow e-beam imaging or by the presence of the e-beam on or near the electrodes.

- Absorption of the e-beam by an electrode could change the average density of transferrable electrons in the material and thereby alter its measured potential.

- The actual area of the electrodes exposed to the liquid can be different from the area probed by the e-beam.

- In experiments with coupled instrumentation, each with different definitions of ground, groundloops are possible and could introduce measurement artefacts.

In order to interpret data coming from these liquid-electrochemical TEM measurement one would ideally need to understand all these potential effects.

In this work, potential sources of measurement artefacts are investigated. The possibility of ground loops and recommendations that minimize them are identified. Offsets in the measured open-circuit potential of working electrodes caused by the presence of the electron beam in TEM are also measured and discussed. Measured potential shifts measured in electrochemical TEM experiments are shown to be largely due to coupling of the beam to components of the cell itself. This work also highlights the possibility of potential measurement offsets when the electrode area exposed to electrolyte is significantly larger than the e-beam illumination area. General criteria are proposed to assist in future in situ liquid-electrochemical cell designs to minimize or eliminate electrochemical measurement artefacts inside a TEM. For example, grounded cells can minimize potential shift artefacts by providing an alternative low impedance discharge path.

These results and recommendations following from this work are the beginning of setting up specific guidelines for best practices in running these experiments and minimize artefacts. They also serve as the start of potential future studies at exploiting the e-beam to induce novel electrochemical phenomenon. 


\section{References:}

[1] M.J. Williamson et al., Nature Materials 2 (2003) p. 532-536.

[2] M. Gu et al., Nano Lett. 13:12 (2013) p. 6106-6112.

[3] R.R. Unocic et al., Microscopy and Microanalysis 20 (2014), p. 1029-1037.

[4] R.L. Sacci et al., Chemical Communications 50 (2014), p. 2104-2107.

[5] J.H. Park et al., Nano Letters 15 (2015), p. 5314-5320.

[6] S.W. Chee et al., Chemical Communications 51 (2015), p. 168-171.

[7] C. Wang, Journal of Materials Research 30 (2015), p 326-339.

[8] J. Lim et al., Science 05 (2016), p. 566-571

[9] The authors acknowledge the Camille Dreyfus Teacher Scholar Award, the Alfred P. Sloan Foundation, and the National Science Foundation (1505635) for support of this research. E.F recognizes a Rackham Predoctoral Fellowship for additional support.

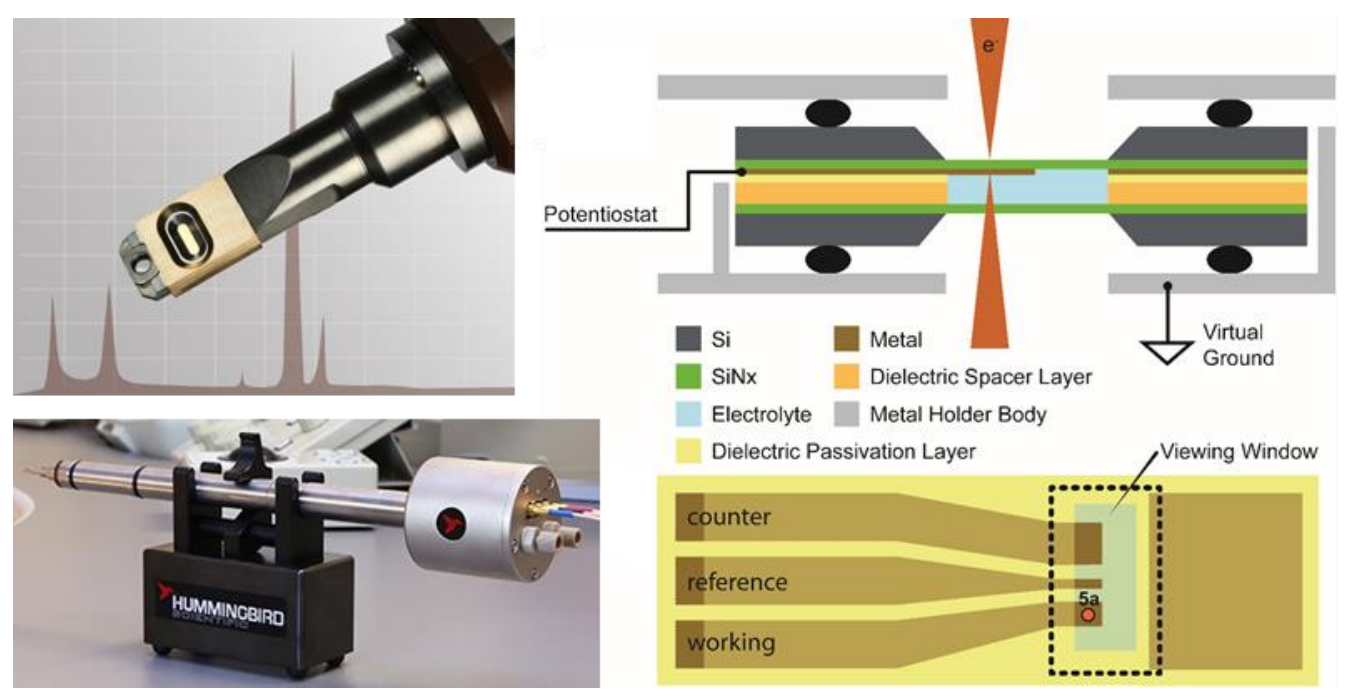

Figure 1. Typical in situ TEM liquid-electrochemical cell setup. Top and Bottom left: liquid cell TEM holder (tip). Top right: vertical cross-section of typical electrochemical setup in the holder tip (several configurations of grounding between the holder, TEM, and potentiostats were compared in this study). Bottom right: schematic representation of a three-electrode setup - electrodes are shown adjacent to each other, but experiments have been conducted with several different electrode layouts (not to scale). 